\title{
El apoyo de estudiantes para la atención de las necesidades educativas: Una estrategia del proyecto UNA educación de calidad de la Universidad Nacional de Costa Rica
}

\section{Student Assistance in Addressing Educational Needs: A Strategy of Quality Education Project of Universidad Nacional, Costa Rica}

\author{
Angélica Fontana Hernández ${ }^{1}$ \\ División de Educación Básica del \\ Centro de Investigación y Docencia en Educación, \\ Universidad Nacional \\ Heredia, Costa Rica \\ angelfontana@ice.co.cr
}

Marcela Sánchez León² Instituto sobre Alcoholismo y Farmacodependencia, Heredia, Costa marcela.sle@gmail.com

Recibido 21 de octubre de 2012 • Corregido 12 de marzo de 2013 • Aceptado 13 de marzo de 2013

1 Master en Pedagogía con énfasis en la Diversidad de Procesos Educativos, Licenciada en Educación Especial con énfasis en Retardo Mental y Bachiller en Problemas de Aprendizaje de la Universidad Nacional de Costa Rica. Académica de la División de Educación Básica del Centro de Investigación y Docencia en Educación (CIDE) de la Universidad Nacional, Costa Rica y responsable del proyecto UNA educación de calidad, que atiende estudiantes con necesidades educativas en su formación profesional. Con experiencia en educación primaria y en los servicios de educación especial. Tiene publicaciones en el campo de la pedagogía en el ámbito nacional e internacional.

2 Licenciada en Psicología de la Universidad Nacional, Costa Rica. Con experiencia en educación primaria sobre la prevención de sustancias psicoactivas del Instituto sobre Alcoholismo y Farmacodependencia del Ministerio de Salud. Tiene publicaciones en el campo de la psicología en el ámbito nacional. 
URL: http://www.una.ac.cr/educare

CORREO: educare@una.cr

Resumen. En 1998 se crea, en la Universidad Nacional, el proyecto UNA educación de calidad, el cual proporciona atención directa a estudiantes que presentan una condición de discapacidad o necesidades educativas en su formación profesional. De acuerdo con la experiencia generada desde el quehacer del proyecto en el contexto universitario, en los años 2008 y 2010, el equipo de trabajo tomó la decisión de valorar las labores desarrolladas con el grupo de estudiantes de apoyo, para conocer los alcances en la atención proporcionada y promover los cambios requeridos. Este proceso se llevó a cabo mediante la modalidad de talleres, la cual permitía integrar la actuación de cada participante, tanto en forma individual como en grupo y en diferentes momentos. Se utilizaron guías de trabajo en grupo, con sesiones de discusión sobre el concepto de necesidades educativas y de análisis de las formas de apoyo utilizadas; un instrumento con frases incompletas; se presentó un video alusivo a la temática abordada, y se finalizó con una discusión sobre los sentimientos y emociones generadas durante las labores de apoyo. Entre los principales hallazgos se destaca la diversificación de las formas de apoyo desplegadas por cada estudiante para atender las necesidades educativas de sus pares, según las condiciones personales y sociales de cada uno, así como de la diversificación de los espacios de interacción e intercambio construidos, lo cual les permitió, a ambos grupos de estudiantes, una reconstrucción del concepto tradicional del término de discapacidad y un mayor desarrollo de las habilidades y destrezas personales y sociales para su permanencia en el contexto universitario, asimismo para su inclusión social y laboral. Se presentan recomendaciones en la atención a la población estudiantil que participa del Proyecto, particularmente en la diversificación de las formas de apoyo y seguimiento, siendo un desafío para el quehacer cotidiano del proyecto UNA educación de calidad.

Palabras claves. Formas de apoyo, necesidades educativas, desarrollo integral, educación inclusiva, Costa Rica, Revista Electrónica Educare.

Abstract. The Quality Education Project of the Universidad Nacional, Costa Rica (UNA Educación de Calidad) was created in 1998 as a direct response channel for students with disabilities or educational needs in their professional training. Considering the experience generated throughout the project's activity at the University in 2008 and 2010, the project team decided to evaluate the work that had been developed with the assistance of a group of students, in order to identify the results of services provided and make the appropriate changes. This process was implemented through workshops, which enabled the group to observe the performance of each participant, individually and collectively, at different times. During the workshops, group work guides were used in the discussion sessions on educational needs and assistance methods, an instrument with incomplete phrases was applied, a video on the issue being discussed was shown, and a final analysis was made on the feelings and emotions that emerged from the assistance activities. Among the main findings, this study points out a range of assistance methods developed by each student to respond to the educational needs of his/her peers, based on the personal and social conditions of each one of them; in addition to a number of spaces created for exchange and interaction. This helped both groups of students to work in the reconstruction of the traditional concept that is associated to the term of "disability" and further develop personal abilities and skills that will contribute to the students' permanence in the university, as well as to their social and labor inclusion. The study provides useful recommendations to meet the needs of the student population participating in the Project, particularly in terms of follow-up and assistance methods, a challenge to be undertaken in the everyday activity of UNA Educación de Calidad.

Keywords. Assistance methods, educational needs, comprehensive development, inclusive education. 


\author{
El que da recibe, \\ el que siembra cosecha, \\ pero el que brinda apoyo \\ siempre tiene una mano amiga \\ con quien compartir
}

A. Fontana

En el año 1998 inicia el proyecto UNA educación de calidad para todos los estudiantes y las estudiantes de la Universidad Nacional, código: 010341. Este nace como una iniciativa de la División de Educación Básica (DEB) del Centro de Investigación en Docencia y Educación (CIDE) para ofrecer un servicio de apoyo a un grupo de estudiantes, matriculado en distintas carreras de la Universidad Nacional (UNA), y que presentaba una discapacidad.

Como referencia importante para respaldar la necesidad de crear este proyecto se tomó en cuenta lo establecido en la legislación vigente en el ámbito internacional, tales como: La declaración mundial sobre educación para todos (UNESCO, 1990), Normas uniformes sobre igualdad de oportunidades para las personas con discapacidad (ONU, 1993), La conferencia mundial sobre necesidades educativas especiales: Acceso y calidad (UNESCO, 1994), Convención internacional para la eliminación de todas las formas de discriminación contra las personas con discapacidad (OEA, 1999), Marco de acción de Dakar. Educación para todos: Cumplir nuestros compromisos comunes (UNESCO, 2000), Declaración de Cochabamba (UNESCO, 2001) y la Convención de los Derechos Humanos de las personas con discapacidad (ONU, 2006). Toda esta legislación tiene el propósito de orientar el establecimiento de leyes y políticas nacionales en los países miembros de la ONU que velan por el cumplimiento de los Derechos Humanos de la personas, especialmente, aquellas en condición de discapacidad.

Respecto a la legislación nacional, se consideró: la Ley 7600 Igualdad de oportunidades para las personas con discapacidad (Costa Rica, Asamblea Legislativa, 1996) y la Directriz 27: Garantizar el cumplimiento de los derechos de las personas con discapacidad y mejorar su calidad de vida (Ministerio de la Presidencia, 2001), la Convención de los Derechos Humanos de las personas con discapacidad (Costa Rica, Asamblea Legislativa, 2008). Estas leyes han impulsado cambios en la organización de las instituciones educativas, tanto en el ámbito administrativo como curricular. Por lo anterior, desde el quehacer del proyecto se proporciona atención directa a este grupo de la diversidad, cumpliendo, de esta forma, con los principios de justicia y solidaridad que rigen el quehacer de la Universidad Nacional, según el Estatuto Orgánico (Universidad Nacional, 1993). 
URL: http://www.una.ac.cr/educare

CORREO: educare@una.cr

\section{Referentes conceptuales sobre la atención de las necesidades educativas y las formas de apoyo}

El abordaje pedagógico de la integración educativa se fundamentó en la filosofía de la normalización y en los principios de integración, sectorización e individualización (León, 1999.)

Por consiguiente, la persona con una condición de discapacidad es percibida como perteneciente al colectivo, con derechos individuales y sociales que garantizan su participación plena en las oportunidades, los bienes y los servicios de la sociedad, especialmente, en el ámbito educativo. Es decir, a recibir atención pedagógica en el contexto más cercano, asimismo, a que se le adaptaran los procesos educativos a sus capacidades, ritmos y estilos de aprendizaje.

En este sentido, Arnaiz (2003) indica que la educación debe ser vista como un proceso dinámico y flexible, el cual permite que las personas reconozcan su semejanza con los otros y acepten su diversidad personal mediante la interacción con el colectivo al que pertenecen.

Desde esta perspectiva, las necesidades educativas se encuentran en estrecha relación con las ayudas pedagógicas o servicios de apoyo educativo requeridos por el grupo de estudiantes en su formación educativa. Por esto, la presencia de necesidades educativas en un estudiante o una estudiante es una condición más de sus características personales, lo cual no la define como persona. La actitud de respeto y comprensión que los actores educativos ${ }^{3}$ manifiesten a su individualidad permite que cada estudiante sea reconocido como un ser integral, con diferencias individuales y sociales. Por tanto, las necesidades educativas pueden surgir por múltiples causas. Estas pueden ser por condiciones personales del grupo de estudiantes, tales como, deficiencias físicas, sensoriales o mentales; por su forma particular de aprender, o bien por la interrelación de sus particularidades con su entorno social: familia, escuela o comunidad (CENAREC, 2005) que limitan su desarrollo y aprendizaje. También, se pueden derivar de los estilos de enseñanza que no consideran las condiciones de aprendizaje ni las adaptaciones necesarias para el estudiantado, así como la falta de productos de apoyo (recursos tecnológicos y ayudas técnicas).

Un aspecto importante de resaltar es que, actualmente, el concepto de necesidades educativas es entendido desde una perspectiva más amplia y flexible que permite incorporar las diferentes conceptualizaciones presentadas en los foros y declaraciones mundiales organizadas por la ONU y la UNESCO. Estas incluyen tanto aspectos personales como socioculturales del estudiantado, con el fin de garantizar una educación de calidad para todos los grupos que conforman la sociedad, sin ningún tipo de discriminación ni exclusión.

3 Se entiende por actores educativos como todos los miembros de la comunidad educativa conformado por el personal docente, y administrativo, la población estudiantil y los padres de familia que participan en forma activa en los procesos de enseñanza y de aprendizaje. 
Por lo anterior, las necesidades educativas percibidas y valoradas desde este nuevo enfoque generan un cambio de concepción, en el sentido de que no solo las personas con una discapacidad pueden presentar condiciones específicas en su formación educativa; se asume que estas pueden surgir por diversos factores tanto personales como socioculturales, es decir, se amplía su carácter de aplicación a todos los grupos de la sociedad.

Por otra parte, las necesidades educativas tienen dos características significativas (Sánchez y Torres, 2002): la primera enfatiza el carácter interactivo, es decir, la necesidad se define en relación con el contexto social en que se desenvuelve y no solo dependen de las condiciones personales del estudiante. La segunda característica es la dimensión relativa de la necesidad, es decir, esta está determinada por un tiempo y un espacio, por tanto, no puede tener un carácter definitivo o permanente. En este sentido, Zúñiga (2002) expresa que al asumir este carácter interactivo, las necesidades educativas del grupo de estudiantes, tiene, entonces, un carácter relativo en su abordaje pedagógico.

Otro aspecto que se desprende de esta reflexión es que el concepto de necesidades educativas simplifica la relación entre las distintas modalidades y niveles del sistema educativo, porque asume que la necesidad es un aspecto inherente a la condición humana y todas las personas, en algún momento de su vida, requieren de apoyos para responder a las exigencias del desarrollo personal y del contexto social.

Asumir el concepto de necesidades educativas dentro del abordaje pedagógico de la integración educativa permite que el objeto de la educación especial se oriente hacia las diferentes situaciones de aprendizaje y no en las condiciones personales del grupo de estudiantes (Aguilar, 2004), las cuales se han empleado para etiquetar y clasificar y, peor aún, para discriminar y excluir.

En este sentido, Sánchezy Torres (2002) indican que la concreción curricular debe circunscribirse en un proyecto curricular de centro, el cual debe favorecer a todos los actores educativos involucrados, con el fin de alcanzar una educación de calidad de acuerdo con las posibilidades de los centros educativos y las características de la población estudiantil; es decir, un proyecto educativo que contemple estrategias de atención a la diversidad estudiantil y que incorpore contenidos y objetivos comunes, así como, la formación permanente de la comunidad educativa.

De acuerdo con la experiencia en la atención de las necesidades educativas, se evidenció la importancia de realizar diversos ajustes en los elementos del entorno educativo, con el fin de garantizar las condiciones de accesibilidad a la población estudiantil, particularmente a la que presentaba una condición de discapacidad u otras condiciones personales y sociales que le generaban condiciones específicas para aprender en su formación educativa. Debido a esto se introduce el concepto de adecuaciones curriculares, definidas por Puigdellivol (2007 p. 97) como "(...) las adaptaciones y ayudas para la asimilación del currículo y las acomodaciones de este a las necesidades educativas"; asimismo, Borsani (2003) define las AC como las modificaciones específicas que realiza el personal docente para responder a situaciones personales que presenta el grupo de estudiantes en el contexto educativo. Entre estas, se pueden mencionar los ajustes a las actividades, a los materiales empleados y a las estrategias de evaluación. 
URL: http://www.una.ac.cr/educare

CORREO: educare@una.cr

Otro tipo de ajuste empleado para responder a las necesidades educativas de la población estudiantil son las adecuaciones de acceso al currículo (en adelante, AAC), las cuales no se consideran dentro del ámbito curricular propiamente, ya que son más adaptaciones o modificaciones que preparan el entorno educativo y que les permite a los estudiantes acceder al currículo general, según sus características y necesidades.

De acuerdo con Sánchez y Torres (2002), las AAC son aquellas modificaciones en los elementos del entorno que van a permitir a los estudiantes acceder al currículo. Entre estas se pueden citar:

1. La adaptación de los elementos humanos del centro educativo, con el fin de establecer estrategias de trabajo en equipo y facilitar la atención educativa del estudiantado. Algunos ejemplos son la agrupación flexible de estudiantes, y la formación de equipos técnicos de coordinación, de orientación o de apoyo educativo.

2. Las adaptaciones en los espacios yaspectosfísicos parafacilitar el uso yel desplazamientoen las instalaciones del centro educativo, como la eliminación de las barreras arquitectónicas, la disposición del mobiliario, la señalización y otros ajustes de accesibilidad.

3. Las adaptaciones en los equipamientos y recursos con el fin de proveer diversidad de materiales que responda a las necesidades de los estudiantes.

4. Las adaptaciones en el tiempo según las actividades que se realizan en el centro educativo.

Según Puigdellivol (2007) las AAC son las modificaciones que se realizan en el contexto educativo. Estas se agrupan en tres categorías, a saber:

1. Las adecuaciones arquitectónicas y ambientales: se relacionan con la eliminación de las barreras en la infraestructura o el espacio físico que limitan el desplazamiento y la movilidad autónoma.

2. Las adecuaciones organizativas: se refieren a todas aquellas medidas de orden y disposición del entorno educativo que se requieren, según las necesidades educativas.

3. Las adecuaciones didácticas: consideran las acciones que tienen que ver con la presentación de los recursos didácticos utilizados en los procesos de enseñanza y de aprendizaje.

También, las AAC se refieren a "(...) las modificaciones o adaptaciones en el currículo necesarias para compensar o contrarrestar diferencias de aprendizaje en los estudiantes, de acuerdo con sus particularidades, las cuales, pueden ser temporales o permanentes" (CENAREC, 2005, p. 5). 
Retomando las conceptuaciones de las AAC, anteriormente expuestas, se puede apreciar que las diferentes agrupaciones presentadas se enfocan en distintos aspectos, según la visión de los autores; no obstante, su propósito siempre es crear las condiciones de accesibilidad en el entorno educativo para facilitar a todos los estudiantes y las estudiantes el acceso al ámbito curricular y al contexto social.

\section{Etapas del proyecto UNA educación de calidad en el contexto de la Universidad Nacional}

En la formulación de la primera etapa del proyecto (1998-2010), se contaba con la participación de dos profesionales en educación especial y estudiantado becado que realizaba horas asistente $(\mathrm{HA})^{4}$ y horas colaboración $(\mathrm{HC})^{5}$, quienes coadyuvaban en las labores administrativas y en la atención individual del grupo de estudiantes participantes.

Si bien esta iniciativa se encontraba adscrita a la DEB del CIDE, en su marco de acción, desde el año 2005, el proyecto realiza funciones a nivel institucional y proporciona atención directa a los estudiantes y a las estudiantes de la institución que presentaban condiciones personales y sociales que incidían en su formación profesional. Se integran, de esta forma, los siguientes principios del Modelo pedagógico (UNA, 2008) el respeto a la diversidad en todas sus expresiones y el compromiso con la igualdad de oportunidades para la construcción de una sociedad más justa (Fontana y Vargas, 2005).

Esta primera etapa estuvo fundamentada en la perspectiva teórica y jurídica descrita anteriormente, por lo que este referente justificó cada una de las acciones realizadas en la atención de las necesidades educativas, las cuales se atendían desde las deficiencias cognitivas, sensoriales (visual y auditiva) y físico-motora que presentaba el grupo de estudiantes, generadas por una condición de discapacidad y otras condiciones personales y sociales, así como por las dificultades en el aprendizaje, en la atención y en la concentración.

Por tanto, las diversas formas de apoyo que se desplegaban en el proyecto tenían el propósito de compensar las diferencias individuales del grupo de estudiantes participantes, para que pudiera acceder a su formación profesional mediante la intervención de diferentes profesionales. Acorde con ello, una de las principales estrategias usadas fue la adaptación de los recursos y materiales que posibilitaran el aprendizaje, con el fin de que cada estudiante pudiera cursar el plan de estudios de sus carreras respectivas. Por tanto, los ajustes curriculares y los productos de apoyo resultaban las formas más utilizadas (Fontana y Vargas, 2007).

Con el fin de considerar un concepto amplio de las AAC, desde el proyecto se asumió la concepción que indica qué son las adaptaciones, las modificaciones o los ajustes en los

$4 \quad$ Estudiante becado por la Universidad Nacional y avanzado en su carrera que realiza diferentes labores en la unidades académicas, departamentos, proyectos y programas con una retribución económica.

5 Estudiante becado por la Universidad Nacional y avanzado en su carrera que realiza diferentes labores en la unidades académicas, departamentos, proyectos y programas sin una retribución económica. 
URL: http://www.una.ac.cr/educare

CORREO: educare@una.cr

elementos del entorno educativo -recursos y materiales didácticos, espacio físico, tiempo y factor humano- las que facilitan, a la población estudiantil, el acceso al currículo y su permanencia en el contexto educativo (Fontana, Espinoza y León, 2009). Estos ajustes estaban determinados por las acciones de distribución, organización y provisión que realizaban los actores educativos a estos elementos del entorno educativo, con el fin de generar las condiciones de accesibilidad para cada estudiante, especialmente a quienes presentaban condiciones de discapacidad.

Es importante indicar que las AAC tenían un papel determinante en la vida cotidiana del grupo de estudiantes con discapacidad, porque les permitía una participación autónoma en los diferentes contextos, particularmente, en el área académica, así como mejorar su calidad de vida y favorecer su inserción tanto social como laboral.

Por otra parte, dentro de las AAC, la provisión de las ayudas técnicas (programas de cómputo especializados, sillas de rueda, audífonos, anteojos, entro otros) para las personas en condición de discapacidad constituían un aspecto relevante, pues son los productos de apoyo que les permite, en primera instancia, desplazarse y movilizarse en el contexto educativo en forma independiente, así como una participación plena en condiciones de igualdad y equidad en el contexto universitario (Fontana y Rodríguez, 2010).

\section{El valioso apoyo de estudiantes en la atención de las necesidades educativas en el contexto universitario}

Un aspecto que ha permitido una mejor organización y funcionamiento del proyecto, consiste en el apoyo del grupo de estudiantes que realizan horas colaboración $(\mathrm{HC})$ en el contexto universitario, el cual está establecido en el Manual de organización de la Vicerrectoría de Vida Estudiantil (VVE) de la UNA, en el I Capítulo De los fundamentos del sistema de becas y su organización, como un principio de colaboración de la población estudiantil que recibe una beca en la universidad, por asistir y apoyar en programas, proyectos y actividades académicas que se ejecuten en el ámbito académico, por considerarse importantes espacios para su desarrollo personal y profesional.

En este mismo manual, en el IV Capítulo de la beca Luis Felipe González Flores y en el V Capítulo de la beca Omar Dengo, entre los requisitos que se establecen para que los estudiantes y las estudiantes puedan obtener este tipo de beca se indica, en el Artículo 40 inciso f, lo siguiente:

Colaborar $\mathbf{8}^{6}$ horas por semana, dedicadas a labores académicas o paraacadémicas. El cumplimiento de las horas, será establecido de mutuo acuerdo entre [sic] la o el estudiante y la o el responsable. El incumplimiento de este requisito, será sancionado

6 La cantidad de horas colaboración que deben realizar los estudiantes y las estudiantes, se ejecutará hasta tanto la institución realice un diagnóstico del aprovechamiento de este apoyo en los programas, proyectos y actividades académicas de la institución; asimismo se establezca un perfil de su desempeño según el Transitorio 1 del Manuel de Organización de Funciones de la Vicerrectoría de Vida Estudiantil. 
según lo establecido en el numeral 129 de este Manual. Se reconocerá y documentará en el historial académico como una actividad cocurricular. La ubicación de [sic] las y los estudiantes para que realicen sus horas de colaboración, será establecida en coordinación con las Unidades Académicas y el Departamento de Bienestar Estudiantil. La responsabilidad de la asignación de tareas, el control y el seguimiento lo realizará la o el funcionario [sic] designado para tal efecto por las unidades académicas u otras instancias universitarias, donde se ubiquen estudiantes para el cumplimiento de horas colaboración. (Universidad Nacional, 2012 p. 15)

También en el VII Capítulo de los tipos de becas y beneficios por rendimiento académico en el Artículo 58 sobre la beca a estudiante asistente, se indica que es un estímulo que se otorga a estudiantes destacados académicamente que posean aptitudes y destrezas comprobadas para colaborar y desempeñarse en áreas de interés institucional; asimismo, en el Artículo 59.1, se establece la colaboración del estudiante asistente en actividades académicas, programas o proyectos de la institución según sus capacidades, por lo cual se le otorga la exención total del pago de los créditos y un aporte económico mensual, definido de acuerdo con los criterios aprobados por el Consejo de Becas. Se reconocerá y documentará en el historial académico como una actividad cocurricular.

Por lo anterior, el proyecto desde sus inicios cuenta con el apoyo de estudiantes que realizan $\mathrm{HA}$ y $\mathrm{HC}$, soporte de suma importancia para el quehacer cotidiano, particularmente para dar atención directa al grupo de estudiantes con necesidades educativas, en el contexto universitario.

Entre las principales formas de apoyo que efectuaba el grupo de estudiantes que realizaban HA y de $\mathrm{HC}$ con su pares con una condición de discapacidad visual se destacan: la lectura oral o el grabado de textos, documentos o libros requeridos en los cursos matriculados; la orientación y la movilidad dentro del contexto universitario cuando era requerida; la digitalización de los documentos escritos, la adaptación de los materiales y recursos (ampliaciones o relieve); el apoyo como referente visual en el salón de clase y en la visita a diferentes lugares fuera de la institución, entre otras.

El grupo de estudiantes que presentaba una condición de sordera, recibía atención de una persona intérprete del lenguaje de señas costarricense (LESCO), cuyo nombramiento se hacía a través de la Vicerrectoría de Vida Estudiantil (VVE). Desde el proyecto, sí así se requería, los estudiantes HC tomaban apuntes en los cursos matriculados.

El grupo de estudiantes que presentaba una condición de discapacidad física, se apoyaba con la digitalización de los trabajos y en el estudio individual; asimismo las barreras relacionadas con el acceso al espacio físico y a la infraestructura eran referidas a las unidades académicas (UA) o al Área de Planeamiento Espacial de la institución para que se construyeran los ajustes requeridos, tales como rampas con sus barandas de soporte, servicios sanitarios adaptados, ascensores en los edificios de uso cotidiano en el contexto universitario (la 
URL: http://www.una.ac.cr/educare

CORREO: educare@una.cr

biblioteca, facultades y centros). No obstante, este esfuerzo dio frutos a partir del año 2007, cuando se apreció un lento crecimiento en las condiciones de accesibilidad en el contexto universitario (Fontana y Vargas, 2007).

El grupo de estudiantes que presentaban necesidades educativas generadas por otras condiciones personales y sociales (dificultades en el aprendizaje, en la atención y la concentración) demandaban, principalmente, apoyo de los estudiantes y las estudiantes que realizaban $\mathrm{HC}$ para el estudio individual, refuerzo en el área de inglés y matemática, así como para la digitación de trabajos.

Con este panorama, en el año 2008 el equipo del proyecto tomó la decisión de realizar acciones de valoración del trabajo desarrollado con el grupo de estudiantes que realizan HC, para conocer los alcances de la atención proporcionada y promover los cambios necesarios.

A continuación se presenta las principales características de la estrategia metodológica propuesta.

\section{Estrategia metodológica}

La dinámica cotidiana que se despliega en el interior del proyecto se circunscribe desde un enfoque constructivista (Solé y Coll, 2007), en el cual los miembros del equipo de trabajo presentan sus propuestas, se discuten y analizan de acuerdo con la realidad social imperante para establecer las prioridades de acción en el contexto universitario.

Por tanto, se tomó la decisión de emplear la técnica de trabajos grupales para recabar la información requerida en este proceso de valoración. De acuerdo con Barrantes (2000, p. 217), esta se refiere a "(...) un conjunto de procedimientos que se utilizan para lograr con eficiencia las metas propuestas (...)". Lo anterior, con el fin de facilitarle al grupo de estudiantes que realizan $\mathrm{HC}$ distintos espacios de discusión, reflexión y construcción de conocimientos sobre la atención a las necesidades educativas; identificar las situaciones que limitaban la interacción y el intercambio entre el grupo de participantes, así como, la construcción de nuevos aprendizajes para buscar las alternativas de solución a dichas situaciones.

El proceso de valoración se llevó a cabo mediante la modalidad de talleres, la cual permite integrar la actuación de los participantes y las participantes en diferentes actividades, tanto en forma individual como en grupo y en diferentes momentos.

\section{Primera fase de talleres: Explorando para construir}

Esta primera fase de talleres se realizó en el mes de abril del año 2008, con el objetivo general de promover un espacio para que el grupo de estudiantes que realizaban $\mathrm{HC}$ en el proyecto UNA educación de calidad expresara sus vivencias y conocimientos adquiridos en relación con el apoyo que le proporcionaba al grupo de estudiantes con necesidades educativas. 
Más específicamente se pretendía: Describir las formas de apoyo que proporcionaba este grupo de estudiantes de $\mathrm{HC}$ al grupo de participantes del proyecto; identificar los aspectos que limitaban u obstaculizaban su desempeño; conocer las emociones y sentimientos surgidos durante sus labores de apoyo, e identificar los aprendizajes construidos en esta experiencia.

Los talleres tenían una metodología participativa, en donde el aporte de cada participante era fundamental para alcanzar los objetivos propuestos. De manera resumida, las actividades desarrolladas fueron las siguientes: se inició con una dinámica de presentación, luego se realizó una discusión en forma grupal sobre el concepto de necesidades educativas, las formas de apoyo utilizadas, las limitaciones y los obstáculos encontrados, asimismo los aprendizajes construidos. Posteriormente se completó un instrumento con frases incompletas, se presentó un video alusivo a la temática abordada y se concluyó con una discusión sobre los sentimientos y emociones que emergieron durante las labores de apoyo.

Se realizaron 7 talleres con una duración de 1 hora y media a 2 horas. Se contó con la participación de 26 estudiantes ( 23 mujeres y 3 hombres), los cuales se agruparon en cuatro horarios diferentes, según su disponibilidad. Si por alguna situación alguna persona del grupo de estudiantes no podía presentarse en el horario establecido, se realizaban las actividades propuestas en forma individual o en parejas.

Es importante indicar que en la realización de los talleres las estudiantes que realizaban $\mathrm{HA}$ en el proyecto contribuyeron significativamente en el desarrollo de estos: planificaron los objetivos y sesiones, elaboraron materiales, alistaron el equipo, aplicaron algunas actividades y asistieron a las académicas en el desarrollo de las actividades propuestas (Fontana, Vargas y Holts, 2008).

\section{Segunda fase de talleres: Construyendo nuevos caminos}

Esta segunda fase de talleres se realizó en el mes de agosto del 2010, orientada por el objetivo general de facilitar un espacio para que el grupo de estudiantes que realizaban $\mathrm{HC}$ en el proyecto discutieran sobre las funciones y las responsabilidad asumidas en la labores de apoyo al grupo con necesidades educativas.

En términos más concretos se buscaba: describir las funciones y las responsabilidades de este grupo de estudiantes; determinar los logros alcanzados en su labor de apoyo; identificar las dificultades enfrentadas, y conocer las recomendaciones para mejorar su colaboración y para darles seguimiento.

Estos talleres mantuvieron la metodología participativa. Las actividades realizadas fueron las siguientes: se inició con una dinámica de presentación del proyecto, luego se realizó una discusión en forma grupal sobre la importancia del apoyo proporcionado, las funciones y las responsabilidades tanto de los estudiantes y las estudiantes que realizan $\mathrm{HC}$ como de quienes reciben el apoyo. Posteriormente, se conversó acerca de los logros alcanzados durante este 
URL: http://www.una.ac.cr/educare

CORREO: educare@una.cr

periodo y las dificultades que enfrentan en las labores de apoyo. Al finalizar, se completó un instrumento de evaluación de la actividad y se conversó acerca de las recomendaciones para mejorar el apoyo y el seguimiento al grupo de estudiantes que realizan $\mathrm{HC}$ en el proyecto, asimismo se entregaron desplegables informativos con recomendaciones generales para proporcionar apoyo a una persona con discapacidad en el contexto universitario.

Se realizaron 3 talleres con una duración de 1 hora y media a 2 horas, se contó con la participación de 12 estudiantes ( 9 mujeres y 3 hombres), los cuales se agruparon en 3 horarios diferentes, según la disponibilidad de cada participante. Si por alguna situación alguien no podía presentarse en el horario establecido se atendían en forma individual o en parejas.

Como siempre, en la realización de los talleres se contó con la colaboración de los estudiantes que realizaban $\mathrm{HA}$ en el proyecto, quienes contribuyen en la elaboración de materiales, uso del equipo y asistencia a las académicas en la aplicación de las actividades propuestas (Fontana y Rodríguez, 2010).

\section{Reconstrucción y análisis de los resultados en los talleres}

El objetivo de esta etapa consistió en recopilar la información recabada en cada uno de los momentos vivenciados en los talleres, se llevó a cabo mediante una sistematización y análisis de la información obtenida en cada taller, según las actividades desarrolladas.

Primera fase de talleres. Al inicio de la primera fase de los talleres en el 2008 y de acuerdo con lo expresado por el grupo de estudiantes que realizaban $\mathrm{HC}$, asimismo al observar el video, se evidenciaron ideas y pensamientos sobre la valoración y conciencia de las capacidades de las personas con una condición de discapacidad, o bien con otras condiciones asociadas a situaciones personales y sociales. También se observó la interiorización de las diferencias entre el concepto de discapacidad física-motora y cognitiva. Esto quizá se explica por la experiencia generada en la interacción con el grupo de estudiantes que participaba del proyecto y a experiencias personales previas con esta población. También se apreció un reconocimiento de la importancia de su apoyo en la vida cotidiana de cada uno de ellos y ellas.

Los apoyos brindados al grupo de estudiantes con necesidades educativas permitían que estos pudieran acceder al proceso educativo, puesto que promovía la participación en las actividades propuestas en los salones de clase, una mayor interacción con el grupo de compañeros, así como una mayor comprensión de los temas observados, lo cual se reflejaba en el rendimiento académico. Entre los apoyos brindados destacaban: la adaptación de materiales, el apoyo como referente visual durante las clases, actividades extracurriculares, toma de apuntes, explicaciones a personas con sordera cuando el intérprete no llegaba, entre otras acciones. 
También los apoyos proporcionados favorecieron la interacción social entre pares, en ocasiones se evidenció que, debido a la relación de amistad construida entre ambos grupos de estudiantes, este apoyo se extendió a situaciones personales y a actividades recreativas en el contexto universitario. El grupo de estudiantes que realizaban $\mathrm{HC}$ tenía clara la trascendencia del apoyo que brindaban, al ser conscientes de que esto contribuía a generar condiciones de accesibilidad para los estudiantes y las estudiantes que participan del proyecto, ya que, de esta forma, podían responder a las exigencias del sistema de formación en el ámbito universitario.

También, aquel grupo de estudiantes que realizaban $\mathrm{HC}$ con estudiantes con discapacidad visual, resaltó el trabajo en equipo realizado -aclaran que la iniciativa era de ellos y ellas, y que en ningún momento confundían la labor requerida con el hacerles los trabajos, ya que se respetaba la capacidad de cada persona-.

Por otro lado, un grupo de estudiantes afirmó que no tenían claro hasta dónde llegaba su responsabilidad o bien que esta tarea había sido difícil. Es necesario tener presente este aspecto en las inducciones realizadas al inicio de cada año, pues este constituye un elemento esencial y cotidiano en sus labores de apoyo.

Las dificultades mencionadas por este grupo de estudiantes de $\mathrm{HC}$ se puede presentar por factores. Tal es el caso de la manipulación que pueden ejercer quienes presentan necesidades educativas para que les efectúen sus tareas y trabajos, o bien por la falta de información sobre las responsabilidades de cada persona. Esto se relaciona con la importancia del desarrollo de la independencia, lo cual puede promover un avance en el ámbito universitario. Núñez (2007) resalta que en las personas con discapacidad las figuras de tránsito, que son aquellas en las que las personas con discapacidad pueden apoyarse para afrontar ámbitos externos a su hogar, son fundamentales, por lo que en la dinámica interna del proyecto, el grupo de estudiantes de HC cumple esta función de tránsito, fortaleciendo el desarrollo de la independencia, aspecto fundamental para la preparación destinada al ámbito laboral.

Un aspecto que se destaca es que las estudiantes y los estudiantes de HC mencionan que, a partir de la observación de las habilidades de las personas con discapacidad visual, han dejado de realizar ciertas labores debido a la habilidad desarrollada por ellos y ellas en tareas tales como: elaborar resúmenes sobre temas abordados en clase y sacar ideas principales.

Es importante recalcar que el grupo de estudiantes de HC es consciente de la importancia de la colaboración que brindan, y la han asumido con responsabilidad y honestidad, al notar que su cumplimiento es fundamental para el rendimiento académico de las personas apoyadas. Esto también se pudo notar por los sentimientos positivos expresados en relación con su labor y en las relaciones de amistad construidas.

Entre las limitaciones y obstáculos presentes durante el cumplimiento de las $\mathrm{HC}$, se resaltaron el desconocimiento, por parte del personal académico, así como del grupo de compañeros del estudiante que presenta necesidades educativas, sobre el papel que se debe asumir en la aplicación de los ajustes curriculares. Esto en ocasiones confluye con otra de 
URL: http://www.una.ac.cr/educare

CORREO: educare@una.cr

las limitaciones mencionadas, la falta de tiempo debido a la carga académica del grupo de estudiantes de HC, e incide no solo en su labor académica, sino también con el desempeño de las personas apoyadas, pues no siempre disponen del tiempo suficiente para atenderles. Esto se agrava cuando las personas con necesidades educativas solicitan material con poco tiempo de anticipación y genera tensión en el grupo de estudiantes de HC.

Otra de las limitantes que influyen negativamente es la falta y condiciones del equipo tecnológico existente en la Sala Adaptada con Tecnología de Apoyo (SATA) ubicada en la Biblioteca Joaquín García Monge. Se evidenció cierto disgusto por el equipo, por el poco mantenimiento que este recibe por parte del personal de Referencia de la Biblioteca, así como la carencia de apoyo técnico cuando tienen problemas. Lo anterior pone en evidencia la importancia de promover mejoras en las condiciones tanto ambientales como de equipo tecnológico para que el grupo de estudiantes de $\mathrm{HC}$ se desempeñe de la mejor manera posible, favoreciendo la integración de un grupo de la diversidad como lo son aquellos estudiantes y aquellas estudiantes con discapacidad que se encuentran matriculadas en la Universidad Nacional.

Otro factor relacionado con el uso del equipo tecnológico es que la mayoría manifestó la necesidad de conocer el manejo adecuado al iniciar el desarrollo de las HC, ya que esto incide en la calidad de su trabajo; pues de lo contrario deben aprender por sí mismos, y esto perjudica el proceso educativo ya que se retrasa las labores de asistencia.

Por otra parte, un elemento que facilitó las labores del grupo de estudiantes al realizar HC, y que además beneficia su propia formación universitaria, consistió en apoyar a estudiantes que cursaban las mismas materias que ellas o ellos matricularon, o bien, que se encuentren en la misma carrera. Esto permitió alivianar la sobrecarga, pues las actividades que realizan son las mismas que ellos y ellas deben cumplir.

Es importante recalcar que las limitaciones y obstáculos mencionados en ningún momento promueven visiones de incapacidad de las personas con necesidades educativas, sino más bien han logrado percibir las capacidades de las personas inscritas en el proyecto. A pesar de ello, en ciertos momentos han sentido incertidumbre con respecto al trato que se les debe dar en el contexto universitario, por el poco conocimiento sobre las características de la discapacidad de la persona a quien apoyan.

Las relaciones que establecieron despertaron el interés en algunos y algunas, en indagar sobre esta temática en sus trabajos finales de graduación, a su vez, mencionaron sentimientos de respeto, comprensión, solidaridad, tolerancia y admiración hacia estos estudiantes, así como valoración de sus cualidades humanas: un ejemplo de referencia para su crecimiento personal.

Entre los aprendizajes que el grupo de estudiantes de HC logró se destaca el haber aprendido a tratar a las personas con discapacidad de igual forma que a los demás y lograr construir herramientas personales que facilitaban la interacción. Sin embargo, esto pareciera mostrar cierta ambivalencia, debido que un grupo de estudiantes manifestó que 
el desconocimiento de las características de las personas con discapacidad les generaba incertidumbre con respecto al trato adecuado que debía dárseles, pues tenían dudas generadas por la convivencia al realizar HC.

La presencia de sentimientos como aprecio y respeto evidenció una sensibilización para con las personas que presentan una condición de discapacidad; sin embargo, aunque este aspecto es fundamental, se debe fomentar un mayor aprendizaje y comprensión sobre el temática de la persona en condición de discapacidad, para realizar un apoyo adecuado a este grupo de estudiantes.

Otro de los aprendizajes importantes logrados es que la mayoría estaban claros de las habilidades de las personas a quienes apoyaban. Esto evidenció, a su vez, el cambio en las concepciones acerca de las personas con discapacidad, los cuales han dejado de centrarse en las deficiencias y las limitaciones, rescatando las capacidades que poseen y partir de estas para promover su desarrollo integral, tal y como Núñez (2007) afirma al decir que es necesario dejar atrás paradigmas que "(...) han sesgado la mirada focalizándose en lo patológico, en las fallas, sin abrirnos el horizonte para ver las capacidades, recursos y fortalezas de las personas expuestas a situaciones adversas (p. 214).

En este sentido, el grupo de participantes manifestó que, antes de haber compartido con quienes apoyan, pensaban que las personas con discapacidad eran diferentes, por lo que les provocaba temor el hecho de saber que iban a trabajar con una de ellas, caracterizando esta labor como difícil. A pesar de ello, los sentimientos expresados en cuanto al trabajo llevado a cabo evidenció agrado y satisfacción por la labor realizada, la cual valoraron de forma positiva, pues esta experiencia les permitió aprender sobre esta temática y transformó las visiones que tenían con respecto al concepto de discapacidad y a las capacidades que presentan las personas en esta condición. Es evidente el compromiso de parte del grupo de estudiantes de $\mathrm{HC}$, pues concluyen su labor a pesar de la presencia de limitaciones que generan sentimientos de malestar, tales como, frustración, enojo, tensión, estrés, cólera, entre otros.

También, la labor realizada por el grupo de $\mathrm{HC}$ ha tenido impacto en el desarrollo personal de cada una y cada uno, debido a que la mayoría afirmó que han logrado ser más expresivos, responsables y esforzados, lo cual, evidencia un impacto al cumplir un requisito universitario, objetivo explícito del principio de colaboración de la población estudiantil, según el Manual de organización de funciones de la Vicerrectoría de Vida Estudiantil (Universidad Nacional, 2012).

Segunda fase de los talleres. En el año 2010, el grupo de estudiantes que realizó HC indicó que logró, a nivel personal, un mayor conocimiento sobre las características de las personas con discapacidad física- motora y visual, lo cual le permitió relacionarse de una forma adecuada, en las labores de apoyo y en las relaciones de amistad construidas.

Otro aspecto mencionando por el grupo de $\mathrm{HC}$, es que el estudiantado con discapacidad a quien apoyaba mostró mayor autonomía e independencia en el desempeño, tanto en el ámbito personal como académico; esta condición se evidenció o en su participación en las 
URL: http://www.una.ac.cr/educare

CORREO: educare@una.cr

sesiones de clase y en la elaboración de los trabajos. Lo anterior, posiblemente se debía a que labores de apoyo proporcionadas por el grupo de estudiantes de $\mathrm{HC}$ se focalizaron en el desarrollo de las destrezas básicas para mejorar el rendimiento académico del estudiante a quien se apoya.

Otro aprendizaje alcanzado por los estudiantes y las estudiantes de $\mathrm{HC}$ es un mayor conocimiento en el uso de equipo tecnológico, tales como programas de computación especializados (lector de pantalla JAWS, habilitadores de texto Abby Fine Reader entre otros), amplificadores de textos e imágenes para personas con discapacidad visual, lo cual favoreció tanto su formación profesional como la del grupo de estudiantes a quienes le proporcionaban apoyo. Es decir, las labores realizadas desde el proyecto facilitaron un mutuo aprendizaje entre el grupo de estudiante que realizan $\mathrm{HC}$ y el grupo de participantes, con un intercambio de conocimientos y un desarrollo de habilidades en forma espontánea y natural.

Por lo anterior, la mayoría de participantes expresan que el apoyo proporcionado por el equipo del proyecto constituye un soporte fundamental para el grupo de estudiantes que presentan una condición de discapacidad, ya que sin este soporte su adaptación y permanencia en el contexto universitario se encontraría coartada.

Otro aspecto que se distinguió es la satisfacción personal de parte del grupo de HC con relación con las labores de apoyo, ya que valoraron estas acciones de forma positiva y consideraron que los buenos resultados obtenidos son producto del trabajo conjunto de ambos grupos de estudiantes. No obstante, una parte de ellos y ellas opina que la experiencia de apoyar un estudiante con discapacidad ha sido un poco difícil ya que le exigió un mayor compromiso, responsabilidad y disposición por aprender nuevos conocimientos para poder apoyar a este grupo de estudiantes.

Entre las dificultades que enfrentó el grupo de participantes en sus labores de apoyo mencionaron los problemas en el uso adecuado del equipo de tecnológico, ya que si un programa de computación deja de funcionar, se tiene que esperar a que llegue un estudiante que realizaba $\mathrm{HA}$ en el proyecto o el técnico de informática del CIDE para que revisara el equipo, desinstalara e instalara de nuevo los programas requeridos. Este tiempo de espera limita sus labores de apoyo y genera sentimientos de malestar (frustración, enojo, impotencia), porque no se logra un avance en el desarrollo del trabajo asignado.

Otro aspecto que generó sentimientos de malestar entre el grupo de estudiantes de apoyo, era la corrección de los textos digitalizados con anterioridad, asimismo, la descripción de las figuras, gráficos, cuadros y tablas en estos documentos, porque se requiere mucha paciencia y dedicación para lograr un resultado adecuado.

En este punto, también indicaron que se han presentado problemas con el acceso al equipo tecnológico (computadoras y digitalizadores) en la sala de la biblioteca Joaquín García Monge, debido a que coinciden varios estudiantes con discapacidad visual y uno o dos estudiantes de apoyo, y el espacio disponible en los cubículos es reducido. 
Otro aspecto que le generó dificultades al grupo de estudiantes de apoyo fue la digitalización de grabaciones de clases, entrevistas y charlas, porque se requiere destrezas de escucha y de mecanografía para lograr un avance el trabajo, de lo contrario se debe dedicar mucho tiempo para concluirlo.

Además, el grupo de estudiantes de HC indicó que la coordinación con los estudiantes y las estudiantes a quienes debían apoyar para entregar el material elaborado y la disponibilidad, según el horario de cada ciclo lectivo, eran aspectos que causaban conflictos, ya que en algunas ocasiones estos no coincidían y las formas de comunicación utilizadas no eran las más efectivas (mensajes de voz, llamadas telefónicas). Asimismo, mencionó que, en ciertas situaciones (enfermedad) tenían que desplazarse a los lugares de residencia para entregar el material que requerían para los cursos, lo cual implica una erogación económica y una inversión de tiempo no contemplada en las labores de apoyo. Lo anterior puso en evidencia que el esfuerzo y el compromiso asumido por el grupo de estudiantes de HC trascendían el contexto universitario.

Por último expresó que un elemento generador de conflictos era la creencia, de parte de algunos estudiantes y algunas estudiantes que recibían apoyo, de que se les debía hacer todo y les atribuyeron funciones al grupo de estudiantes que realizaban $\mathrm{HC}$ que no les correspondían (elaborar y concluir trabajos). Lo anterior, probablemente se deba a los patrones de crianza directivos de sus familias y de las instituciones educativas donde se han formado, que les restringía las oportunidades de tomar decisiones en su vida, por lo que dejaban, a las personas con una condición de discapacidad, en un estado de indefensión. Según Meléndez (2002) esta es una indefensión aprendida del medio social, el cual, influyó en la construcción de su autoconcepto como personas con limitaciones y con pocas habilidades de autodeterminación y autogestión, la cual se refleja en su comportamiento, particularmente, en las reacciones de vulnerabilidad para adaptarse a la sociedad en que se encuentran inmersas, o bien para establecer metas para sus vidas.

\section{Sugerencias para mejorar el trabajo desplegado en el proyecto UNA educación de calidad}

Entre las sugerencias que aportó el grupo de estudiantes que realizan $\mathrm{HC}$, se mencionaron:

- Realizar reuniones periódicas para abordar situaciones específicas en las labores de apoyo (irresponsabilidad, asignación de labores que no corresponden).

- Facilitar información relacionada con la atención de las necesidades educativas en el contexto universitario.

- Mejorar el uso del recurso tecnológico e instalación de programas de computación especializados.

- Valorar el proceso desarrollado e identificar los aspectos que se deben mejorar, específicamente, el seguimiento y el control del apoyo proporcionado. 
URL: http://www.una.ac.cr/educare

CORREO: educare@una.cr

También indicaron que es necesario mejorar el orden en que se está realizando la digitalización de los documentos, en particular, señalar claramente dónde se finalizó para iniciar en forma adecuada.

Un elemento que se resalta son las palabras de motivación y aliento para el equipo del proyecto UNA educación de calidad por parte los estudiantes y las estudiantes participantes en los talleres. Estas reflejan una apreciación muy positiva del impacto que se despliega cotidianamente con las labores de apoyo. Algunos ejemplos son los siguientes:

¡Excelente, sigan asíi El proyecto es excelente tanto para el que realiza horas colaboración como para el estudiante que requiere apoyo por el buen trabajo que se realiza. ¡Sigan adelante, los felicito! El estudiante que realiza horas colaboración que viene al proyecto aprende a ser mejor persona cada día. ¡Buen trabajo por las personas con discapacidad! (Fontana y Sánchez, 2008, p. 33)

\section{Reflexiones finales y recomendaciones}

A continuación se presenta las reflexiones finales, las cuales retoman las ideas relevantes del trabajo desarrollado en los talleres en cada una de las fases.

1. Las diversas formas de apoyo realizadas por el grupo de estudiantes que realizan HC resalta la importancia de estas acciones en el desempeño del grupo de estudiantes que participa del proyecto UNA educación de calidad, particularmente porque permite el desarrollo de mayores habilidades y destrezas personales y sociales, requeridas para integrase y adaptarse a la dinámica universitaria.

2. Los sentimientos de incertidumbre sobre el trato que se les debe proporcionar a las personas con una condición de discapacidad por parte del grupo de estudiantes que realizan labores de apoyo en el Proyecto, pone en evidencia que la historia de segregación y diferenciación social en la atención educativa, en la cual han estado inmersos ambos grupos, limita la adquisición de las destrezas básicas para establecer relaciones interpersonales en forma adecuada.

3. En la aplicación de los ajustes curriculares y en la determinación de los apoyos por parte del personal académico y administrativo de las unidades académicas, el estudiantado tiene un papel fundamental, porque es la persona responsable no solo de velar el cumplimiento de sus derechos según la legislación nacional vigente, sino de promover una concienciación en los miembros de la comunidad universitaria sobre el valor de diversidad y su inclusión social. 
4. Los diferentes espacios de interacción e intercambio entre el grupo estudiantes que realizan labores de apoyo y el grupo de estudiantes que participan del proyecto, particularmente, en condición de discapacidad, han permitido una reconstrucción de la visión tradicional sobre la personas con discapacidad, logrando posicionarse en una perspectiva centrada en las cualidades y talentos, que les ha permitido desenvolverse en forma exitosa en diferentes contextos sociales y alcanzar sus metas.

5. Las diversas labores de apoyo realizadas por el grupo de estudiantes con las personas que participan del proyecto ha permitido, en ambos grupos, un intercambio de conocimientos y experiencia en el ámbito personal y social, situación que ha impactado positivamente en la formación profesional de cada participante, al promover, de esta forma, una transformación social, pues se logra la aceptación de las diferencias como parte de la condición humana.

6. El trabajo colaborativo desplegado entre el personal académico, el grupo de estudiantes de apoyo y el personal del proyecto permitió un mayor desempeño académico de los estudiantes y las estudiantes, puesto que las funciones de los diferentes actores involucrados se articularon y se complementaron para dar respuesta a las diferentes situaciones que emergieron durante el desarrollo de los cursos.

7. Es importante reforzar los espacios disponibles en la Universidad Nacional, así como contar con mayor cantidad de equipos como grabadoras, computadoras, escáneres y programa JAWS, para que quienes apoyan el proyecto puedan realizar sus labores de forma ágil, y lograr, así, el impacto en el rendimiento educativo del grupo con discapacidad.

8. Aunado a lo anterior, es fundamental capacitar al grupo de estudiantes que realizan $\mathrm{HC}$ en el proyecto, en el manejo de los productos de apoyo para las personas con discapacidad visual, así como aclarar las dudas generadas en la atención proporcionada, tales como estrategias para desplazarse con una persona que presenta una condición de ceguera, conocimientos básicos de la cultura de la población sorda, formas de apoyo para una persona que utiliza una andadera para movilizarse, entre otras. Esta información es valiosa y debe tenerse en cuenta a la hora de interaccionar con una persona con discapacidad, pues su desconocimiento, al proporcionarle apoyo, genera sentimientos de incertidumbre sobre cómo darle un trato adecuado.

9. Es necesario llevar a cabo procesos de reflexión el grupo de estudiantes que presentan necesidades educativas, con el fin de promover una participación activa en la definición de los a poyos y en la aplicación de las adecuaciones curriculares. De esta forma se afianzan sus derechos y deberes; asimismo se propicia un adecuado rendimiento académico y una mayor autonomía e independencia en el contexto universitario.

10. Es esencial escuchar las necesidades que presentan tanto el grupo de estudiantes que se benefician de los servicios que ofrece el proyecto como del grupo que lo apoya, con el fin de hacer efectivas las recomendaciones que proporcionan, para fomentar una 
URL: http://www.una.ac.cr/educare

CORREO: educare@una.cr

mejor labor de las personas que realizan $\mathrm{HC}$, así como un rendimiento adecuado de los estudiantes y las estudiantes.

11. Por último, es fundamental propiciar una mayor comunicación y vinculación entre los responsables de los programas, proyectos y actividades académicas de la Universidad Nacional, para desplegar una labor articulada en el apoyo al estudiantado que presenta una condición de discapacidad o necesidades educativas, así como para lograr su inclusión efectiva en el contexto universitario, en condiciones de igualdad y equidad según las legislación vigente en el país (Fontana, 2012).

\section{Referencias}

Aguilar, G. (julio, 2004). Del exterminio a la educación inclusiva: Una visión desde la discapacidad. Conferencia presentada en el V Congreso Educativo Internacional: De la educación tradicional a la educación inclusiva. Heredia: Universidad Interamericana de Costa Rica.

Arnaiz, P. (2003). Educación inclusiva: Una escuela para todos. España: Ediciones Aljibe.

Barrantes, R. (2000). Investigación: Un camino al conocimiento, un enfoque cualitativo y cuantitativo. San José, Costa Rica: EUNED.

Borsani, M. J. (2003). Adecuaciones curriculares. Apuntes de atención a la diversidad._Buenos Aires: Novedades Educativas.

Centro Nacional de Recursos para la Inclusión Educativa [CENAREC] (2005). Adecuaciones de acceso al currículo. Inducción a equipos de investigación. San José, Costa Rica: Manuscrito inédito.

Costa Rica, Asamblea Legislativa. (29 de mayo de 1996). Igualdad de oportunidades para las personas con discapacidad (Ley N 7600). La Gaceta. Diario Oficial N 112. San José: Imprenta Nacional.

Costa Rica. Asamblea Legislativa. (29 de setiembre del 2008). Ley № 8661: Convención sobre los derechos humanos de las personas con discapacidad. La Gaceta Diario Oficial $N^{\circ} 187$. San José, Costa Rica: Imprenta Nacional.

Costa Rica, Ministerio de la Presidencia. (1 de enero de 2001). Directriz $N^{\circ} 27$. Garantizar el cumplimiento de los derechos de las personas con discapacidad y mejorar su calidad de vida. Decreto Ejecutivo No. 30843-MP-MIVAH-MIDEPLAN. Recuperado de http://www.cnree. go.cr/images/documentos/legislacion/Directriz27Cimad.pdf

Fontana, A. (mayo-junio, 2012). Redes de apoyo en atención de la diversidad: aspectos críticos y e implicaciones en el contexto de la Universidad Nacional de Costa Rica: $1^{\text {a }}$ parte. Visión Docente Con-Ciencia, 11(64). Recuperado de http://www.ceuarkos.com/Vision_docente/ costarica64.pdf 
Fontana, A. y Vargas. M. (2005). Informe anual del proyecto UNA educación de calidad para todos los estudiantes y las estudiantes de la Universidad Nacional ( $\mathrm{N}^{\circ}$ 010341). Heredia: Vicerrectoría Académica, Universidad Nacional. Manuscrito inédito.

Fontana, A. y Vargas. M. (2007). Informe anual del proyecto UNA educación de calidad para todos los estudiantes y las estudiantes de la Universidad Nacional ( $\mathrm{N}^{\circ}$ 010341). Heredia: Vicerrectoría Académica, Universidad Nacional. Manuscrito inédito.

Fontana, A., Vargas. M. y Holts, B. (2008). Informe anual del proyecto UNA educación de calidad para todos los estudiantes y las estudiantes de la Universidad Nacional ( $\left.N^{\circ} 010341\right)$. Heredia: Vicerrectoría Académica, Universidad Nacional. Manuscrito inédito.

Fontana, A., Espinoza, A. y León, H. (2009). Implementación de las adecuaciones de acceso en las instituciones educativas costarricenses. San José, Costa Rica: CENAREC.

Fontana, A. y Sánchez, M. (2008). Informe de los talleres con el grupo de estudiantes que realizan horas colaboración en el proyecto UNA educación de calidad. Heredia, Centro de Investigación en Docencia y Educación, Universidad Nacional. Manuscrito inédito.

Fontana, A. y Rodríguez, R. (2010). Informe anual del proyecto UNA educación de calidad para todos los estudiantes y las estudiantes de la Universidad Nacional ( $\mathrm{N}^{\circ}$ 010341). Heredia: Vicerrectoría Académica, Universidad Nacional. Manuscrito inédito.

León, M. J. (1999). De la integración escolar a la escuela inclusiva o escuela para todos. (Capítulo segundo) En M. Á., Lou y N. López (Coords.), Bases psicopedagógicas de la educación especial (pp. 39- 61). España: Ediciones Pirámide.

Meléndez, L. (2002). La inclusión escolar del alumno con discapacidad intelectual. Bogotá: GLARRIIPP.

Núñez, B. (2007). Familia y discapacidad. De la vida cotidiana a la teoría. Argentina: Lugar Editorial.

Organización de Estados Americanos (OEA). (1999). Convención internacional para la eliminación de todas las formas de discriminación contra las personas con discapacidad. Recuperado de http://www.cnree.go.cr/sobre-discapacidad/legislacion/44-convencion-interamericanapara-la-eliminacion-de-todas-las-formas-de-discriminacion-contra-las-personas-condiscapacidad.html

Organización de las Naciones Unidas [ONU]. (20 de diciembre, 1993). Normas uniformes sobre la igualdad de oportunidades para personas con discapacidad. Nueva York. Recuperado de http://www.cnree.go.cr/sobre-discapacidad/legislacion/41-normas-uniformes-sobre-laigualdad-de-oportunidades-para-personas-con-discapacidad.html

Organización de las Naciones Unidas (ONU). (13 de diciembre, 2006). Convención sobre los derechos de las personas con discapacidad. Recuperado de http://www.un.org/spanish/ disabilities/documents/gid/conventionfaq.pdf 
URL: http://www.una.ac.cr/educare

CORREO: educare@una.cr

Organización de las Naciones Unidas para la Educación, la Ciencia y la Cultura (UNESCO). (5-9 de marzo, 1990). Declaración mundial sobre educación para todos "Satisfacción de las necesidades básica de aprendizaje". Celebrado en Jomtien, Tailandia. Recuperado de http://www.oei.es/efa2000jomtien.htm

Organización de las Naciones Unidas para la Educación, la Ciencia y la Cultura [UNESCO]. (10 de junio, 1994). Declaración de Salamanca. Conferencia mundial sobre necesidades educativas especiales: acceso y calidad. Salamanca, España. Recuperado de http://paidos.rediris.es/ genysi/recursos/doc/leyes/dec_sal. htm

Organización de las Naciones Unidas para la Educación la Ciencia y la Cultura (UNESCO). (26-28 de abril, 2000). Marco de acción de Dakar. Educación para todos: Cumplir nuestros compromisos comunes. Dakar, Senegal. Recuperado de http://www.unesco.org/education/efa/ edforall/ dakframspa.shtm

Organización de las Naciones Unidas para la Educación la Ciencia y la Cultura (UNESCO). (5 y 7 de marzo, 2001). Declaración de Cochabamba. Bolivia, Cochabamba. Recuperado de http://unesdoc.unesco.org/images/0012/001214/121485s.pdf

Puigdellivol, I. (2007). La educación especial en la escuela integrada. Una perspectiva desde la diversidad (7a ed.). España: Graó.

Sánchez, A. y Torres, J. A. (2002). Educación especial. Centros educativos y profesores ante la diversidad. Madrid: Ediciones Pirámide.

Solé, I. y Coll, C. (2007) Los profesores y la concepción constructivista. En C. Coll, E. Martín, T. Mauri, M. Miras, J. Onrubia, I. Solé y A. Zabala (Auts.), El constructivismo en el aula (pp. 7-23) (18a Ed.). Barcelona: Graó. Recuperado de http://books.google.co.cr/books?id=BzOef9UI Db4C\&printsec=frontcover\&hl=es\&source=gbs_ge_summary_r\&cad=0\#v=onepage\&q\& $\mathrm{f}=$ false

Universidad Nacional (UNA). (1993). Estatuto Orgánico. Heredia: Costa Rica: Autor. Recuperado de http://www.juridica.una.ac.cr/index.php?option=com_remository\&ltemid=0\&func=st artdown\&id=144

Universidad Nacional (UNA). (2008) Modelo pedagógico de la Universidad Nacional. Recuperado de http://www.una.ac.cr//index.php?option=com_remository\&ltemid=0\&func=startdow n\&id=141

Universidad Nacional (UNA). (15 de febrero, 2012). Manual de organización de funciones de la Vicerrectoría de Vida Estudiantil (R-3827-2011 del 8 de diciembre del 2011). UNA-Gaceta $N^{\circ}$ 2. Recuperado de http://www.juridica.una.ac.cr/index.php?option=com_remository\&lte mid $=0$ \&func $=$ fileinfo\&id $=384$ 
URL: http://www.una.ac.cr/educare

Zúñiga, E. (2002). Las adecuaciones curriculares en la universidad: Un derecho y una responsabilidad impostergables. En R. Jiménez (ed.), Las personas con discapacidad en la educación superior. Una propuesta para la diversidad e igualdad. (pp. 141-153. San José: Fundación Justicia y Género.

\section{Cómo citar este artículo en APA:}

Fontana, A. y Sánchez, M. (2013). El apoyo de estudiantes para la atención de las necesidades educativas: Una estrategia del proyecto UNA educación de calidad de la Universidad Nacional de Costa Rica. Revista Electrónica Educare, 17(2), 31-53. Recuperado de http:// www.revistas.una.ac.cr/index.php/EDUCARE/issue/current

Nota: Para citar este artículo en otros sistemas puede consultar el hipervínculo "Como citar el artículo" en la barra derecha de nuestro sitio web:

http://www.revistas.una.ac.cr/index.php/EDUCARE/index 